\title{
Analysis of some interior point continuous trajectories for convex programming
}

\author{
Xun Qian* Li-Zhi Liao ${ }^{\dagger}$ and Jie Sun ${ }^{\ddagger}$
}

\begin{abstract}
In this paper, we analyze three interior point continuous trajectories for convex programming with general linear constraints. The three continuous trajectories are derived from the primaldual path-following method, the primal-dual affine scaling method, and the central path, respectively. Theoretical properties of the three interior point continuous trajectories are fully studied. The optimality and convergence of all three interior point continuous trajectories are obtained for any interior feasible point under some mild conditions. In particular, with proper choice of some parameters, the convergence for all three interior point continuous trajectories does not require the strict complementarity or the analyticity of the objective function. These results are new in the literature.
\end{abstract}

Keywords: continuous trajectory, interior point method, convex programming, ordinary differential equation

AMS Subject Classification: 34D23, 90C25, 90C51

\section{Introduction}

Consider the following linearly constrained convex programming problem

$$
\begin{array}{ll}
\min & f(x) \\
\text { s.t. } & A x=b, x \geq 0,
\end{array}
$$

where $f(x)$ is a smooth convex function, $b \in R^{m}$, and $A$ is an $m \times n$ matrix with full row rank, $m<n$. As a blanket assumption, we assume that the optimal value for problem $(\mathrm{P})$ is finite and attainable, therefore, we use min rather than inf in problem $(\mathrm{P})$.

The following notations are used in the sequel

$$
\begin{array}{cl}
R_{+}^{n}=\left\{x \in R^{n} \mid x \geq 0\right\}, & R_{++}^{n}=\left\{x \in R^{n} \mid x>0\right\}, \\
\mathcal{P}^{+}=\left\{x \in R^{n} \mid A x=b, x \geq 0\right\}, & \mathcal{P}^{++}=\left\{x \in R^{n} \mid A x=b, x>0\right\} .
\end{array}
$$

\footnotetext{
${ }^{*}$ Department of Mathematics, Hong Kong Baptist University, Kowloon Tong, Kowloon, Hong Kong, P.R.C. Email: 13479784@life.hkbu.edu.hk

${ }^{\dagger}$ Corresponding author. Department of Mathematics, Hong Kong Baptist University, Kowloon Tong, Kowloon, Hong Kong, P.R.C. Email: liliao@hkbu.edu.hk. The work of L.-Z. Liao was supported in part by grants from Hong Kong Baptist University (FRG) and General Research Fund (GRF) of Hong Kong.

$\ddagger$ Department of Mathematics and Statistics, Curtin University, Australia, Email: jie.sun@curtin.edu.au. The work of J. Sun was supported in part by Australia Research Council under Grant DP-160102918.
} 
It is conventional to assume that $\mathcal{P}^{++}$is nonempty in the analysis of interior point methods. We also assume $f(x) \in C^{3}$ on $R^{n}$.

The Wolfe dual problem [1] associated to $(\mathrm{P})$ is

$$
\begin{array}{cl}
\max & L(y, z)=f(x)-\nabla f(x)^{T} x+b^{T} y \\
\text { s.t. } & -\nabla f(x)+A^{T} y+z=0, z \geq 0,
\end{array}
$$

and the following notations will be used

$$
\begin{aligned}
\mathcal{D}^{+} & =\left\{(x, y, z) \in R^{n} \times R^{m} \times R^{n} \mid-\nabla f(x)+A^{T} y+z=0, z \geq 0\right\}, \\
\mathcal{D}^{++} & =\left\{(x, y, z) \in R^{n} \times R^{m} \times R^{n} \mid-\nabla f(x)+A^{T} y+z=0, z>0\right\} .
\end{aligned}
$$

For linearly constrained convex programming (including linear programming), a number of interior point methods have been proposed, for example, the affine scaling algorithms by Dikin [2], Saigal [3], Tseng and Luo [4], Tsuchiya [5, 6], Gonzaga and Carlos [7], Sun [8], Tseng et al. [9], the primal-dual path-following algorithms by Kojima et al. [10], Monteiro and Adler [11, 12], Renegar [13], Roos [14], Todd and Ye [15], Gonzaga [16], Monteiro [1], Zhu [17], the primal-dual affine scaling algorithms by Monteiro et al. [18], and so on. A survey of search directions in interior point methods for linear programming can be found in Hertog and Roos [19]. Besides the above iterative methods, many continuous trajectories related to the above algorithms are also studied in many different ways, for instance, the affine scaling continuous trajectory by Adler and Monteiro [20], Bayer and Lagarias [21], Liao [22], Megiddo and Shub [23], Monteiro [24], the continuous trajectory for a potential reduction algorithm by Monteiro [25], the central path by Mclinden [26], Megiddo [27], Kojima et al. [28], Monteiro and Tsuchiya [29], Vavasis and Ye [30], Witzgall et al. [31], Monteiro [32], Drummond and Svaiter [33]. Some illbehaved central path examples in convex optimization can be found in [34], however these examples are different from problem $(\mathrm{P})$. It should be noted that for linear programming, the primal affine scaling continuous trajectory actually contains the primal central path if the initial point is on the central path.

In the interior point method, the central path plays a vital role. For the central path where the barrier function $\sum_{i=1}^{n}-\ln x_{i}$ is used in problem $(\mathrm{P})$, convergence can be obtained under the strict complementarity condition [26], the analyticity of $f(x)$ [32], or the condition that there exists a subspace $W$ of $R^{n}$ such that $\operatorname{Ker}\left(\nabla^{2} f(x)\right)=W[33]$.

In this paper, we are interested in the interior point continuous trajectories that are closely related to some interior point methods, specifically, the primal-dual path-following method, and the primal-dual affine scaling method for problem (P). In addition, we will also study a continuous trajectory which is derived from the central path. The primal-dual path-following method is closely related to the central path, however, the search direction in the primal-dual path-following method is actually different from the tangent of the central path. In Section 2, we will study a continuous trajectory which is derived from the search direction in the primaldual path-following method. To our knowledge, this kind of continuous trajectory has not been studied in the literature. The continuous trajectory corresponding to the search direction of the primal-dual affine scaling method will be studied in Section 3. In the linear case, the primal-dual affine scaling continuous trajectory has been studied in [20]. In the nonlinear convex case, since the primal-dual affine 
scaling continuous trajectory is actually a weighted primal-dual central path (see [20] or Theorem 3.3 below), hence part of the results in Section 3 can be regarded as contained in [32]. In Section 4, we will study a direction which is tangent to the primal central path. If the initial point is on the primal central path, the corresponding path is of course the central path, otherwise, we call this path as the central path family. In the linear case, the central path family is actually the primal affine scaling continuous trajectory.

For simplicity, in what follows, $\|\cdot\|$ denotes the 2-norm. $C^{k}$ stands for the class of $k$ th order continuously differentiable functions. Unless otherwise specified, $x_{j}$ denotes the $j$ th component of a vector $x, e$ denotes the column vector of all ones, and $e_{i}$ denotes the unity column vector whose $i$ th component is 1 , the dimensions of $e$ and $e_{i}$ are clear from the context. For any index subset $J \subseteq\{1, \ldots, n\}$, we denote by $x_{J}$ the vector composed of those components of $x \in R^{n}$ indexed by $j \in J$, rank $(Q)$ denotes the rank of the matrix $Q$.

The rest of this paper is organized as follows. In Section 2 - Section 4 , three interior point continuous trajectories, which are closely related to the primal-dual path-following method, the primal-dual affine scaling method, and the central path, will be studied, respectively. In each of these three sections, we divide our discussions into 3 subsections. In Subsection 1, we introduce the corresponding ordinary differential equation (ODE) systems for each interior point continuous trajectory, verify that each ODE system has a unique solution in $\left[t_{0},+\infty\right)$ and show some properties of each continuous trajectory. In Subsection 2, we prove that every accumulation point of the solution of each ODE system is an optimal solution for problem (P) (and problem (D)). In Subsection 3, we show the strong convergence of the solution of each ODE system for any interior feasible point under some mild conditions. Finally, some concluding remarks will be drawn in Section 5 .

\section{The weighted primal-dual path-following continuous trajectory}

In this section, we study a continuous trajectory that is closely related to the primal-dual interior point method. First we define the following sets:

$$
\begin{aligned}
\mathcal{F} & =\left\{(x, y, z) \in R^{n} \times R^{m} \times R^{n} \mid x \in \mathcal{P}^{+},(x, y, z) \in \mathcal{D}^{+}\right\}, \\
\mathcal{F}^{0} & =\left\{(x, y, z) \in R^{n} \times R^{m} \times R^{n} \mid x \in \mathcal{P}^{++},(x, y, z) \in \mathcal{D}^{++}\right\} .
\end{aligned}
$$

The search direction in most of primal-dual interior point algorithms is the solution of the following system:

$$
\left(\begin{array}{ccc}
-\nabla^{2} f(x) A^{T} & I \\
A & 0 & 0 \\
Z & 0 & X
\end{array}\right)\left(\begin{array}{c}
\Delta x \\
\Delta y \\
\Delta z
\end{array}\right)=\left(\begin{array}{c}
0 \\
0 \\
-X Z e+\sigma \mu e
\end{array}\right)
$$

where $\sigma \in[0,1]$ and $\mu=x^{T} z / n$. The search directions of the primal-dual pathfollowing algorithms and primal-dual potential reduction algorithms are generally the solution of system (1). The difference between them is the choice of the stepsize at each iteration. The stepsize in primal-dual path-following algorithms is determined by the neighborhood of the central path, while the stepsize in primal-dual potential reduction algorithms is determined by the potential function. If $\sigma=0$, the search direction is usually called the primal-dual affine scaling direction, and 
for the corresponding continuous trajectory we call it the primal-dual affine scaling continuous trajectory which will be studied in Section 3. If $\sigma=1$ and $(x, y, z) \in \mathcal{F}^{0}$, the search direction is actually the Newton direction of the following equation

$$
\left(\begin{array}{c}
A^{T} y+z \\
A x \\
X Z e
\end{array}\right)=\left(\begin{array}{c}
\nabla f(x) \\
b \\
\mu e
\end{array}\right)
$$

where $\mu=x^{T} z / n$. Evidently, the solution of the above equation is a point on the central path with the dual gap $\mu=\frac{x^{T} z}{n}$, not the optimal solution if $\mu>0$. Therefore in this section we study the search direction with $\sigma \in(0,1)$. We first extend system (1) to a more general form to get the following ODE system

$$
\left\{\begin{array}{l}
-\nabla^{2} f(x) \frac{d x}{d t}+A^{T} \frac{d y}{d t}+\frac{d z}{d t}=0 \\
A \frac{d x}{d t}=0 \\
\gamma_{1} X^{\gamma_{1}-1} Z^{\gamma_{2}} \frac{d x}{d t}+\gamma_{2} X^{\gamma_{1}} Z^{\gamma_{2}-1} \frac{d z}{d t}=-\left(X^{\gamma_{1}} Z^{\gamma_{2}} e-\sigma \mu w\right) \\
\left(x\left(t_{0}\right), y\left(t_{0}\right), z\left(t_{0}\right)=\left(x^{0}, y^{0}, z^{0}\right) \in \mathcal{F}^{0}\right.
\end{array}\right.
$$

where

$$
\begin{aligned}
& t_{0}>0, \sigma \in(0,1), \mu=\frac{e^{T} X^{\gamma_{1}} Z^{\gamma_{2}} e}{n}, \gamma_{1}>0, \gamma_{2}>0, w \in R_{++}^{n}, \sum_{i=1}^{n} w_{i}=n, \\
& x \in R_{++}^{n}, X=\operatorname{diag}(x) \in R^{n \times n}, z \in R_{++}^{n}, Z=\operatorname{diag}(z) \in R^{n \times n} .
\end{aligned}
$$

We call this ODE system the weighted primal-dual path-following ODE system. The unique (Theorem 2.3) solution of ODE system (2) defines the weighted primaldual path-following continuous trajectory for problem $(\mathrm{P})$. The condition that the weighted vector $w$ satisfies $\sum_{i=1}^{n} w_{i}=n$ is to guarantee $\mu=\frac{e^{T} X^{\gamma_{1}} Z^{\gamma_{2} e}}{e^{T} w}=\frac{e^{T} X^{\gamma_{1}} Z^{\gamma_{2}} e}{n}$. From the above ODE system, we can get two equivalent explicit forms, one is

$$
\left\{\begin{aligned}
\frac{d x}{d t}= & -\frac{1}{\gamma_{1}}\left[I_{n}+\frac{\gamma_{2}}{\gamma_{1}}\left(X Z^{-1}\right)^{\frac{1}{2}} P_{A\left(X Z^{-1}\right)^{\frac{1}{2}}}\left(X Z^{-1}\right)^{\frac{1}{2}} \nabla^{2} f(x)\right]^{-1}\left[X^{1-\gamma_{1}} Z^{-\gamma_{2}}\right. \\
& \left.-Z^{-1} X A^{T}\left(A Z^{-1} X A^{T}\right)^{-1} A X^{1-\gamma_{1}} Z^{-\gamma_{2}}\right]\left(X^{\gamma_{1}} Z^{\gamma_{2}} e-\sigma \mu w\right), \\
\frac{d y}{d t}= & \frac{1}{\gamma_{2}}\left(A Z^{-1} X A^{T}\right)^{-1} A X^{1-\gamma_{1}} Z^{-\gamma_{2}}\left(X^{\gamma_{1}} Z^{\gamma_{2}} e-\sigma \mu w\right) \\
& +\left(A Z^{-1} X A^{T}\right)^{-1} A X Z^{-1} \nabla^{2} f(x) \frac{d x}{d t} \\
\frac{d z}{d t}= & \nabla^{2} f(x) \frac{d x}{d t}-A^{T} \frac{d y}{d t}, \quad\left(x\left(t_{0}\right), y\left(t_{0}\right), z\left(t_{0}\right)=\left(x^{0}, y^{0}, z^{0}\right) \in \mathcal{F}^{0},\right.
\end{aligned}\right.
$$

where $P_{A\left(X Z^{-1}\right)^{\frac{1}{2}}}=I_{n}-\left(X Z^{-1}\right)^{\frac{1}{2}} A^{T}\left(A Z^{-1} X A^{T}\right)^{-1} A\left(X Z^{-1}\right)^{\frac{1}{2}}, I_{n}$ is the identity matrix of order $n$. The other explicit form is

$$
\left\{\begin{aligned}
\frac{d x}{d t}= & -G\left[X^{1-\gamma_{1}} Z^{1-\gamma_{2}}-\gamma_{2} X A^{T}\left(A G X A^{T}\right)^{-1} A G X^{1-\gamma_{1}} Z^{1-\gamma_{2}}\right] \\
& \left(X^{\gamma_{1}} Z^{\gamma_{2}} e-\sigma \mu w\right), \\
\frac{d y}{d t}= & {\left[\left(A G X A^{T}\right)^{-1} A G X^{1-\gamma_{1}} Z^{1-\gamma_{2}}\right]\left(X^{\gamma_{1}} Z^{\gamma_{2}} e-\sigma \mu w\right), } \\
\frac{d z}{d t}= & \nabla^{2} f(x) \frac{d x}{d t}-A^{T} \frac{d y}{d t}, \quad\left(x\left(t_{0}\right), y\left(t_{0}\right), z\left(t_{0}\right)=\left(x^{0}, y^{0}, z^{0}\right) \in \mathcal{F}^{0},\right.
\end{aligned}\right.
$$

where $G=\left(\gamma_{1} Z+\gamma_{2} X \nabla^{2} f(x)\right)^{-1}$. The following assumption is made throughout Section 2 .

Assumption 1 The set $\mathcal{F}^{0}$ is nonempty. 


\subsection{Properties of the weighted primal-dual path-following continuous trajectory}

First we need the following lemma to obtain the existence and uniqueness of the solution of the weighted primal-dual path-following ODE system (3).

Lemma $2.1\left(A Z^{-1} X A^{T}\right)^{-1} \in C^{1}$ on $R_{++}^{n} \times R_{++}^{n}$ with respect to $x$ and $z$.

Lemma 2.2 If $A, B \in R^{n \times n}$ are both symmetric and positive semidefinite, then all eigenvalues of $A B$ are nonnegative.

Proof. From Corollary 4.6.3 on page 99 in [35], the result is evident.

Theorem 2.3 and Theorem 2.4 below guarantee the existence, uniqueness, and feasibility for the solution of ODE system (3).

THEOREM 2.3 For the weighted primal-dual path-following ODE system (3), there exists a unique solution $(x(t), y(t), z(t))$ with a maximal existence interval $\left[t_{0}, \beta\right)$, in addition, $x(t)>0, z(t)>0$ on the existence interval.

Proof. Since $\left(X Z^{-1}\right)^{\frac{1}{2}} P_{A\left(X Z^{-1}\right)^{\frac{1}{2}}}\left(X Z^{-1}\right)^{\frac{1}{2}}$ and $\nabla^{2} f(x)$ are both symmetric and positive semidefinite, from Lemma 2.2 we know

$$
\left[I+\frac{\gamma_{2}}{\gamma_{1}}\left(X Z^{-1}\right)^{\frac{1}{2}} P_{A\left(X Z^{-1}\right)^{\frac{1}{2}}}\left(X Z^{-1}\right)^{\frac{1}{2}} \nabla^{2} f(x)\right]
$$

is always invertible for any $x>0$ and $z>0$. Furthermore, from $\nabla^{2} f(x) \in C^{1}$ on $R_{+}^{n}, Z^{-1} \in C^{1}$ on $R_{++}^{n}$ with respect to $z$, and Lemma 2.1, it is not hard to see that the right-hand sides of (3) are all locally Lipschitz continuous on $R_{++}^{n} \times R^{m} \times R_{++}^{n}$. Since $R_{++}^{n} \times R^{m} \times R_{++}^{n}$ is an open set, from the Cauchy-Peano theorem and PicardLindelöf theorem, there exists a unique solution $(x(t), y(t), z(t))$ of ODE system (3) on the maximal existence interval $\left[t_{0}, \beta\right)$, for some $\beta>t_{0}$ or $\beta=+\infty$.

Because the right-hand sides of ODE system (3) are defined on the open set $(0,+\infty) \times R_{++}^{n} \times R^{m} \times R_{++}^{n}$, the solution of ODE system (3) is of course in the open set $R_{++}^{n} \times R^{m} \times R_{++}^{n}$, so $x(t)$ and $z(t)$ are both positive on the existence interval. Thus the proof is complete.

Later in this section, it will be shown that $\beta=+\infty$ (Theorem 2.6). To simplify the presentation, in the remaining of this section, $x(t), y(t)$, and $z(t)$ (or $X(t), Z(t)$ ) will be replaced by $x, y$, and $z$ (or $X, Z$ ), respectively, whenever no confusion would occur.

THEOREM 2.4 Let $(x(t), y(t), z(t))$ be the solution of the weighted primal-dual path-following ODE system (3) with the maximal existence interval $\left[t_{0}, \beta\right)$. Then $A x(t)=b$ and $A^{T} y(t)+z(t)=\nabla f(x(t)) \forall t \in\left[t_{0}, \beta\right)$.

Proof. Notice $A^{T} y^{0}+z^{0}=\nabla f\left(x^{0}\right)$ and the first equation in (2), then

$$
A^{T} \frac{d y}{d t}+\frac{d z}{d t} \equiv \nabla^{2} f(x) \frac{d x}{d t}
$$


we have for any $t \in\left[t_{0}, \beta\right)$,

$$
\begin{aligned}
A^{T} y(t)+z(t) & \left.\left.=A^{T}\left(y^{0}+\left.\int_{t_{0}}^{t} \frac{d y}{d t}\right|_{t=\tau}\right) d \tau\right)+z^{0}+\left.\int_{t_{0}}^{t} \frac{d z}{d t}\right|_{t=\tau}\right) d \tau \\
& \left.=A^{T} y^{0}+z^{0}+\left.\int_{t_{0}}^{t}\left(A^{T} \frac{d y}{d t}+\frac{d z}{d t}\right)\right|_{t=\tau}\right) d \tau \\
& \left.=A^{T} y^{0}+z^{0}+\left.\int_{t_{0}}^{t} \nabla^{2} f(x) \frac{d x}{d t}\right|_{t=\tau}\right) d \tau \\
& =\nabla f\left(x^{0}\right)+\nabla f(x(t))-\nabla f\left(x^{0}\right)=\nabla f(x(t)) .
\end{aligned}
$$

From the second equation in (2) and $A x^{0}=b$, we can get $A x(t)=b$ similarly.

Next we show that the solution curve is contained in a bounded set.

THEOREM 2.5 The unique solution $(x(t), y(t), z(t))$ of the weighted primal-dual path-following ODE system (3) is contained in a bounded set in $R_{+}^{n} \times R^{m} \times R_{+}^{n}$, and the bound of $x(t)$ and $z(t)$ depends only on $x^{0}, z^{0}, \gamma_{1}$, and $\gamma_{2}$, the bound of $y(t)$ depends only on $x^{0}, z^{0}, \gamma_{1}, \gamma_{2}, A$, and $f(x)$.

Proof. First we assume that $x(t)^{T} z(t)$ is bounded by $M>0$. By Theorem 2.4 and the convexity of $f(x)$, for any $t \in\left[t_{0}, \beta\right)$

$$
\begin{aligned}
\left(x(t)-x^{0}\right)^{T}\left(z(t)-z^{0}\right) & =\left(x-x^{0}\right)^{T}\left(-A^{T} y+A^{T} y^{0}+\nabla f(x)-\nabla f\left(x^{0}\right)\right) \\
& =\left(A x-A x^{0}\right)^{T}\left(y^{0}-y\right)+\left(x-x^{0}\right)^{T}\left(\nabla f(x)-\nabla f\left(x^{0}\right)\right) \\
& =\left(x-x^{0}\right)^{T}\left(\nabla f(x)-\nabla f\left(x^{0}\right)\right) \geq 0 .
\end{aligned}
$$

Then we get

$$
\left(x^{0}\right)^{T} z(t)+x(t)^{T} z^{0} \leq\left(x^{0}\right)^{T} z^{0}+x(t)^{T} z(t) \leq\left(x^{0}\right)^{T} z^{0}+M .
$$

Since $x(t)>0$ and $z(t)>0$ (Theorem 2.3), we know for any $t \in\left[t_{0}, \beta\right)$ and any $1 \leq i \leq n$,

$$
x(t)_{i} \leq \frac{\left(x^{0}\right)^{T} z^{0}+M}{z_{i}^{0}}, z(t)_{i} \leq \frac{\left(x^{0}\right)^{T} z^{0}+M}{x_{i}^{0}},
$$

therefore $x(t)$ and $z(t)$ are contained in a bounded set in $R_{+}^{n}$, and the bound depends only on $x^{0}, z^{0}$ and $M$.

For $y(t)$, since $A^{T} y(t)+z(t)=\nabla f(x(t))$ and the matrix $A$ is of full row rank which implies $A A^{T}$ is invertible, then we have

$$
y(t)=\left(A A^{T}\right)^{-1} A(\nabla f(x(t))-z(t)) .
$$

Thus $y(t)$ must be contained in a bounded set in $R^{m}$ and the bound depends only on $x^{0}, z^{0}, M, A$, and $f(x)$.

Next we show that there exists a bound $M>0$ which depends only on $x^{0}, z^{0}$, 
$\gamma_{1}$ and $\gamma_{2}$. First from the third equation in (2), we have

$$
\begin{aligned}
\frac{d e^{T} X(t)^{\gamma_{1}} Z(t)^{\gamma_{2}} e}{d t} & =\gamma_{1} e^{T} X^{\gamma_{1}-1} Z^{\gamma_{2}} \frac{d x}{d t}+\gamma_{2} e^{T} X^{\gamma_{1}} Z^{\gamma_{2}-1} \frac{d z}{d t} \\
& =-e^{T}\left(X^{\gamma_{1}} Z^{\gamma_{2}} e-\sigma \mu w\right)=-e^{T} X^{\gamma_{1}} Z^{\gamma_{2}} e+\sigma n \mu \\
& =-(1-\sigma) e^{T} X^{\gamma_{1}} Z^{\gamma_{2}} e,
\end{aligned}
$$

hence

$$
\mu=\mu_{0} e^{-(1-\sigma)\left(t-t_{0}\right)},
$$

where $\mu_{0}=e^{T} X\left(t_{0}\right)^{\gamma_{1}} Z\left(t_{0}\right)^{\gamma_{2}} e / n$.

If $\gamma_{1}>\gamma_{2}$, for any $1 \leq i \leq n$,

$$
x(t)_{i} z(t)_{i}=x_{i} z_{i}^{\frac{\gamma_{2}}{\gamma_{1}}} z_{i}^{\left(1-\frac{\gamma_{2}}{\gamma_{1}}\right)}=\left(x_{i}^{\gamma_{1}} z_{i}^{\gamma_{2}}\right)^{\frac{1}{\gamma_{1}}} z_{i}^{\left(1-\frac{\gamma_{2}}{\gamma_{1}}\right)} \leq(n \mu)^{\frac{1}{\gamma_{1}}} z_{i}^{\left(1-\frac{\gamma_{2}}{\gamma_{1}}\right)}
$$

furthermore,

$$
z(t)_{i} \leq \frac{\left(x^{0}\right)^{T} z^{0}+x(t)^{T} z(t)}{x_{i}^{0}} \leq \frac{\left(x^{0}\right)^{T} z^{0}+x(t)^{T} z(t)}{\min \left(x^{0}\right)}
$$

along with (4), we get

$$
x(t)_{i} z(t)_{i} \leq\left(n \mu_{0}\right)^{\frac{1}{\gamma_{1}}} e^{-(1-\sigma)\left(t-t_{0}\right) / \gamma_{1}}\left[\frac{\left(x^{0}\right)^{T} z^{0}+x(t)^{T} z(t)}{\min \left(x^{0}\right)}\right]^{\left(1-\frac{\gamma_{2}}{\gamma_{1}}\right)},
$$

and

$$
x(t)^{T} z(t) \leq n\left(n \mu_{0}\right)^{\frac{1}{\gamma_{1}}} e^{-(1-\sigma)\left(t-t_{0}\right) / \gamma_{1}}\left[\frac{\left(x^{0}\right)^{T} z^{0}+x(t)^{T} z(t)}{\min \left(x^{0}\right)}\right]^{\left(1-\frac{\gamma_{2}}{\gamma_{1}}\right)} .
$$

Since $0<1-\frac{\gamma_{2}}{\gamma_{1}}<1$, there should exist a constant $M>0$ such that $x(t)^{T} z(t)<M$, and $M$ depends only on $x^{0}, z^{0}, \gamma_{1}$, and $\gamma_{2}$.

For $\gamma_{1}<\gamma_{2}$, we can prove this similarly. For $\gamma_{1}=\gamma_{2}$ and any $1 \leq i \leq n$,

$$
x(t)_{i} z(t)_{i}=\left(x_{i}^{\gamma_{1}} z_{i}^{\gamma_{1}}\right)^{\frac{1}{\gamma_{1}}} \leq\left(n \mu_{0}\right)^{\frac{1}{\gamma_{1}}} e^{-(1-\sigma)\left(t-t_{0}\right) / \gamma_{1}},
$$

hence $x(t)^{T} z(t) \leq n\left(n \mu_{0}\right)^{\frac{1}{\gamma_{1}}} e^{-(1-\sigma)\left(t-t_{0}\right) / \gamma_{1}} \leq n\left(n \mu_{0}\right)^{\frac{1}{\gamma_{1}}}$.

After we get the boundedness of the solution curve, we can extend the existence interval of the solution to infinity.

TheOREM 2.6 Let $(x(t), y(t), z(t))$ be the solution of the weighted primal-dual path-following ODE system (3) with the maximal existence interval $\left[t_{0}, \beta\right)$. Then $\beta=+\infty$. 
Proof. Assume $\beta \neq+\infty$. From Theorem 2.3, we can define

$$
V_{1}(t)=\sum_{i=1}^{n}\left(\gamma_{1} \ln x_{i}+\gamma_{2} \ln z_{i}\right),
$$

then from the third equation in (2), we have

$$
\gamma_{1} X^{-1} \frac{d x}{d t}+\gamma_{2} Z^{-1} \frac{d z}{d t}=-X^{-\gamma_{1}} Z^{-\gamma_{2}}\left(X^{\gamma_{1}} Z^{\gamma_{2}} e-\sigma \mu w\right)
$$

hence

$$
\begin{aligned}
\frac{d V_{1}(t)}{d t} & =e^{T} \gamma_{1} X^{-1} \frac{d x}{d t}+e^{T} \gamma_{2} Z^{-1} \frac{d z}{d t}=-e^{T} X^{-\gamma_{1}} Z^{-\gamma_{2}}\left(X^{\gamma_{1}} Z^{\gamma_{2}} e-\sigma \mu w\right) \\
& =-n+\sigma \mu e^{T} X^{-\gamma_{1}} Z^{-\gamma_{2}} w \geq-n,
\end{aligned}
$$

therefore

$$
V_{1}(t) \geq V_{1}\left(t_{0}\right)-n \beta
$$

But according to the Extension Theorem in $\S 2.5$, [36], we know that the solution $(x(t), y(t), z(t))$ will go to the boundary of the open set $(0,+\infty) \times R_{++}^{n} \times R^{m} \times R_{++}^{n}$. Because of the hypothesis, $\beta \neq+\infty$ and the solution $(x(t), y(t), z(t))$ is bounded (Theorem 2.5), there must exist at least one $i \in\{1, \ldots, n\}$ such that $x_{i}(t) \rightarrow 0$ or $z_{i}(t) \rightarrow 0$ as $t \rightarrow \beta$. In this situation $V_{1}(t)$ will go to $-\infty$ as $t \rightarrow \beta$ since $x(t)$ and $z(t)$ are all bounded (Theorem 2.5), which contradicts (5). Hence the hypothesis is not true and $\beta=+\infty$.

From Theorem 2.5 and Theorem 2.6, we can define the limit set for the solution of the weighted primal-dual path-following ODE system (3). Let $(x(t), y(t), z(t))$ be the solution of ODE system (3), the limit set of $\{x(t), y(t), z(t)\}$ can be defined as follows

$$
\begin{aligned}
\Omega^{1}\left(x^{0}, y^{0}, z^{0}\right)= & \left\{(x, y, z) \mid \exists\left\{t_{k}\right\}_{k=0}^{+\infty} \text { with } \lim _{k \rightarrow+\infty} t_{k}=+\infty\right. \text { such that } \\
& \left.\lim _{k \rightarrow+\infty} x\left(t_{k}\right)=x, \lim _{k \rightarrow+\infty} y\left(t_{k}\right)=y \text { and } \lim _{k \rightarrow+\infty} z\left(t_{k}\right)=z\right\} .
\end{aligned}
$$

TheOREM 2.7 The limit set $\Omega^{1}\left(x^{0}, y^{0}, z^{0}\right)$ is nonempty, compact, and connected. Furthermore $\Omega^{1}\left(x^{0}, y^{0}, z^{0}\right)$ is contained in $\mathcal{F}$.

Proof. From Theorems 2.3, 2.4, and 2.6, we know that the limit set $\Omega^{1}\left(x^{0}, y^{0}, z^{0}\right)$ is contained in $\mathcal{F}$. From Theorem 2.5, we know that the solution $(x(t), y(t), z(t))$ is contained in a bounded closed set. So similar to the proof of Theorem 1.1 on page 390 in [37] (the proof in [37] is for $n=2$, but it can be easily extended to the general case), it can be verified that $\Omega^{1}\left(x^{0}, y^{0}, z^{0}\right)$ is nonempty, compact, and connected.

\subsection{Optimality of the cluster point(s)}

In this subsection, we show that every accumulation point of the solution of the weighted primal-dual path-following ODE system (3) is an optimal solution for 
problems (P) and (D).

TheOREM 2.8 Let $(x(t), y(t), z(t))$ be the unique solution of the weighted primaldual path-following ODE system (3). Then

(i) $x(t)^{T} z(t) \leq L_{2} e^{-(1-\sigma)\left(t-t_{0}\right) / \max \left(\gamma_{1}, \gamma_{2}\right)}$, where $L_{2}>0$ is a constant which depends only on $x^{0}, z^{0}, \gamma_{1}$, and $\gamma_{2}$, hence for any point $\left(x^{1}, y^{1}, z^{1}\right) \in$ $\Omega^{1}\left(x^{0}, y^{0}, z^{0}\right), x^{1}$ is an optimal solution of problem $(P),\left(y^{1}, z^{1}\right)$ is an optimal solution of problem $(D)$.

(ii) For any $1 \leq i \leq n$,

$$
\left(x(t)_{i}^{\gamma_{1}} z(t)_{i}^{\gamma_{2}}-w_{i} \mu\right)=e^{-\left(t-t_{0}\right)}\left(x\left(t_{0}\right)_{i}^{\gamma_{1}} z\left(t_{0}\right)_{i}^{\gamma_{2}}-w_{i} \mu^{0}\right),
$$

where $\mu^{0}=\frac{e^{T} X\left(t_{0}\right)^{\gamma_{1}} Z\left(t_{0}\right)^{\gamma_{2}} e}{n}$.

Proof. Proof of (i). From Theorem 2.5, we know there exists a bound $M>0$ which depends only on $x^{0}, z^{0}, \gamma_{1}$, and $\gamma_{2}$ such that for any $1 \leq i \leq n$,

$$
x(t)_{i} z(t)_{i} \leq M(n \mu)^{\frac{1}{\max \left(\gamma_{1}, \gamma_{2}\right)}},
$$

along with (4), we have

$$
x(t)^{T} z(t) \leq L_{2} e^{-(1-\sigma)\left(t-t_{0}\right) / \max \left(\gamma_{1}, \gamma_{2}\right)},
$$

where $L_{2}=\operatorname{Mn}\left(n \mu_{0}\right)^{\frac{1}{\max \left(\gamma_{1}, \gamma_{2}\right)}}$. Hence for any point $\left(x^{1}, y^{1}, z^{1}\right) \in \Omega^{1}\left(x^{0}, y^{0}, z^{0}\right)$, we have

$$
0 \leq\left(x^{1}\right)^{T} z^{1} \leq \limsup _{t \rightarrow+\infty} x(t)^{T} z(t) \leq 0,
$$

which indicates that $x^{1}$ is an optimal solution of problem $(\mathrm{P})$, and $\left(y^{1}, z^{1}\right)$ is an optimal solution of problem (D).

Proof of (ii). For any $1 \leq i \leq n$, from the third equation in (2), we can get

$$
\frac{d x(t)_{i}^{\gamma_{1}} z(t)_{i}^{\gamma_{2}}}{d t}=-\left(x(t)_{i}^{\gamma_{1}} z(t)_{i}^{\gamma_{2}}-\sigma \mu w_{i}\right)
$$

hence

$$
\frac{d\left(x(t)_{i}^{\gamma_{1}} z(t)_{i}^{\gamma_{2}}-w_{i} \mu\right)}{d t}=-\left(x(t)_{i}^{\gamma_{1}} z(t)_{i}^{\gamma_{2}}-w_{i} \mu\right)
$$

therefore $\left(x(t)_{i}^{\gamma_{1}} z(t)_{i}^{\gamma_{2}}-w_{i} \mu\right)=e^{-\left(t-t_{0}\right)}\left(x\left(t_{0}\right)_{i}^{\gamma_{1}} z\left(t_{0}\right)_{i}^{\gamma_{2}}-w_{i} \mu^{0}\right)$, where $\mu^{0}=$ $\frac{e^{T} X\left(t_{0}\right)^{\gamma_{1}} Z\left(t_{0}\right)^{\gamma_{2} e}}{n}$. Thus the theorem is proved.

\subsection{Convergence of the weighted primal-dual path-following continuous trajectory}

Now, it comes to the key result of Section 3. Under some mild conditions, the solution of the weighted primal-dual path-following ODE system (3) will converge as $t \rightarrow+\infty$. First we give the definition of the analytic center. We define the 
analytic center of a closed convex set $\Omega \subseteq R^{n}$ corresponding to convex function $g(x)$ as the unique minimizer of the following problem:

$$
\begin{array}{ll}
\min & g(x) \\
\text { s.t. } & x \in \Omega \cap \operatorname{dom} g(x),
\end{array}
$$

where dom $g(x)=\{x \mid g(x)<+\infty\}$. Generally, the minimizer of the above problem may not be unique, but in our context the existence and uniqueness of the minimizer can be guaranteed and no confuse would occur. First we need the following two lemmas.

Lemma 2.9 ([38]) Suppose $f$ is differentiable (i.e., its gradient $\nabla f$ exists at each point in dom $f$ ). Then $f$ is convex if and only if dom $f$ is convex and

$$
f(y) \geq f(x)+\nabla f(x)^{T}(y-x)
$$

holds for all $x, y \in \operatorname{domf}$.

LEMma 2.10 If $f(x)$ is convex and analytic, then for any two different optimal solutions $x^{1}$ and $x^{2}$ of problem $(P)$, and any $x \in R^{n},\left(x^{2}-x^{1}\right)^{T} \nabla f(x)=0$.

Proof. Since $f(x)$ is convex, we have for any $\lambda \in[0,1]$,

$$
\begin{aligned}
f\left(x^{1}+\lambda \Delta x\right) & =f\left(\lambda x^{2}+(1-\lambda) x^{1}\right) \\
& \leq \lambda f\left(x^{2}\right)+(1-\lambda) f\left(x^{1}\right) \\
& =f\left(x^{1}\right)=f\left(x^{2}\right),
\end{aligned}
$$

where $\Delta x=x^{2}-x^{1} \neq 0$. Moreover, $x^{1}$ and $x^{2}$ are two different optimal solutions for problem $(\mathrm{P})$ and $\lambda x^{2}+(1-\lambda) x^{1} \in \mathcal{P}^{+}$for any $\lambda \in[0,1]$, hence

$$
f\left(x^{1}+\lambda \Delta x\right)=f\left(x^{1}\right)=f\left(x^{2}\right),
$$

for any $\lambda \in[0,1]$. Since $f(x)$ is analytic, then according to Corollary 1.2.5 in [39], we have for any $\lambda \in R$,

$$
f\left(x^{1}+\lambda \Delta x\right)=f\left(x^{1}\right)=f\left(x^{2}\right) .
$$

By Corollary 8.6.1 of Rockafellar [40], it follows that for any $x \in R^{n}, f(x+\lambda \Delta x)$ will be a constant function of $\lambda \in R$. Hence

$$
\frac{d f(x+\lambda \Delta x)}{d \lambda}=\left(x^{2}-x^{1}\right)^{T} \nabla f(x+\lambda \Delta x)=0,
$$

for any $\lambda \in R$. Let $\lambda=0$ in the above equality, we have for any $x \in R^{n}$, ( $x^{2}-$ $\left.x^{1}\right)^{T} \nabla f(x)=0$. Thus the lemma is proved.

Theorem 2.11 Let $x^{*}$ and $\left(y^{*}, z^{*}\right)$ be optimal solutions for problems $(P)$ and (D), respectively, such that $x^{*}$ and $z^{*}$ have the maximal numbers of positive components among all optimal solutions. Let $(x(t), y(t), z(t))$ be the unique solution of the weighted primal-dual path-following ODE system (3). Then 
(i) for any $1 \leq i \leq n$,

$$
\left(x(t)_{i}^{\gamma_{1}} z(t)_{i}^{\gamma_{2}}-w_{i} \mu\right)=\frac{x\left(t_{0}\right)_{i}^{\gamma_{1}} z\left(t_{0}\right)_{i}^{\gamma_{2}}-w_{i} \mu^{0}}{w_{i} \mu_{0}} e^{-\sigma\left(t-t_{0}\right)} w_{i} \mu .
$$

(ii) a) If $\gamma_{1}<\gamma_{2}$, then $x(t)$ will converge to the analytic center of the optimal solution set of problem $(P)$ corresponding to $-\sum_{x_{i}^{*}>0}\left(w_{i}\right)^{1 / \gamma_{2}}\left(x_{i}\right)^{1-\frac{\gamma_{1}}{\gamma_{2}}}$;

b) if $\gamma_{1}=\gamma_{2}$, and either there exists a pair of primal and dual optimal solutions satisfying the strict complementarity or $f(x)$ is analytic, then $x(t)$ will converge to the analytic center of the optimal solution set of problem $(P)$ corresponding to $-\prod_{x_{i}^{*}>0}\left(x_{i}\right)^{w_{i}^{1 / \gamma_{1}}}$;

c) if $\gamma_{1}>\gamma_{2}$ and $f(x)$ is analytic, then $x(t)$ will converge to the analytic center of the optimal solution set of problem $(P)$ corresponding to $\sum_{x_{i}^{*}>0}\left(w_{i}\right)^{1 / \gamma_{2}}\left(x_{i}\right)^{1-\frac{\gamma_{1}}{\gamma_{2}}}$.

(iii) a) If $\gamma_{1}<\gamma_{2}$, then $z(t)$ will converge to the analytic center of the optimal solution set of problem $(D)$ corresponding to $\sum_{z_{i}^{*}>0}\left(w_{i}\right)^{1 / \gamma_{1}}\left(z_{i}\right)^{1-\frac{\gamma_{2}}{\gamma_{1}}}$;

b) if $\gamma_{1}=\gamma_{2}$, then $z(t)$ will converge to the analytic center of the optimal solution set of problem $(D)$ corresponding to $-\prod_{z_{i}^{*}>0}\left(z_{i}\right)^{w_{i}^{1 / \gamma_{1}}}$;

c) if $\gamma_{1}>\gamma_{2}$, then $z(t)$ will converge to the analytic center of the optimal solution set of problem $(D)$ corresponding to $-\sum_{z_{i}^{*}>0}\left(w_{i}\right)^{1 / \gamma_{1}}\left(z_{i}\right)^{1-\frac{\gamma_{2}}{\gamma_{1}}}$.

Proof. Proof of (i). From Theorem 2.8 and (4), it is straightforward that for any $1 \leq i \leq n$,

$$
\left(x(t)_{i}^{\gamma_{1}} z(t)_{i}^{\gamma_{2}}-w_{i} \mu\right)=c_{i}^{0} e^{-\sigma\left(t-t_{0}\right)} w_{i} \mu=d_{i}^{0}(t) w_{i} \mu,
$$

where $c_{i}^{0}=\frac{x\left(t_{0}\right)_{i}^{\gamma_{1}} z\left(t_{0}\right)_{i}^{\gamma_{2}}-w_{i} \mu^{0}}{w_{i} \mu_{0}}$ and $d_{i}^{0}(t)=c_{i}^{0} e^{-\sigma\left(t-t_{0}\right)}$.

Proof of (ii). If $x^{*}=0$, the result is evident, so we assume $x^{*} \neq 0$ below.

a) If $\gamma_{1}<\gamma_{2}$, from (7), for any $1 \leq i \leq n$,

$$
z(t)_{i}=\frac{\left(w_{i} \mu\left(1+c_{i}^{0} e^{-\sigma\left(t-t_{0}\right)}\right)\right)^{1 / \gamma_{2}}}{x_{i}^{\gamma_{1} / \gamma_{2}}}
$$

then from Theorem 2.4 and Lemma 2.9, we have

$$
\begin{aligned}
0 & \geq f\left(x^{*}\right)-f(x(t)) \geq\left(x^{*}-x(t)\right)^{T} \nabla f(x(t))=\left(x^{*}-x\right)^{T}\left(A^{T} y+z\right) \\
& =\left(x^{*}-x\right)^{T} z=\sum_{i=1}^{n} \frac{\left(w_{i} \mu\left(1+c_{i}^{0} e^{-\sigma\left(t-t_{0}\right)}\right)\right)^{1 / \gamma_{2}}\left(x_{i}^{*}-x_{i}\right)}{x_{i}^{\gamma_{1} / \gamma_{2}}} .
\end{aligned}
$$

This indicates

$$
\sum_{i=1}^{n} \frac{\left(w_{i}\left(1+c_{i}^{0} e^{-\sigma\left(t-t_{0}\right)}\right)\right)^{1 / \gamma_{2}}\left(x_{i}^{*}-x_{i}\right)}{x_{i}^{\gamma_{1} / \gamma_{2}}} \leq 0
$$


then for any accumulation point $\bar{x}$ of $x(t)$, since $x^{*}$ has the maximal number of positive components among all optimal solutions of problem $(\mathrm{P})$ and $\bar{x}$ is also an optimal solution, so for any $i$ with $x_{i}^{*}=0, \bar{x}_{i}=0$ which implies that in (8),

$$
\sum_{x_{i}^{*}=0} \frac{\left(w_{i}\left(1+c_{i}^{0} e^{-\sigma\left(t-t_{0}\right)}\right)\right)^{1 / \gamma_{2}}\left(x_{i}^{*}-x_{i}\right)}{x_{i}^{\gamma_{1} / \gamma_{2}}} \rightarrow-\left(w_{i}\right)^{1 / \gamma_{2}} \bar{x}_{i}^{1-\frac{\gamma_{1}}{\gamma_{2}}}=0
$$

as $x(t) \rightarrow \bar{x}$. Also for any $i$ with $x_{i}^{*}>0, \bar{x}_{i}$ must be positive, since if $\bar{x}_{i}=0$, then

$$
\frac{\left(w_{i}\left(1+c_{i}^{0} e^{-\sigma\left(t-t_{0}\right)}\right)\right)^{1 / \gamma_{2}}\left(x_{i}^{*}-x_{i}\right)}{x_{i}^{\gamma_{1} / \gamma_{2}}} \rightarrow+\infty,
$$

as $x_{i} \rightarrow \bar{x}_{i}$ which contradicts with (8). Hence by (8), $\bar{x}$ must have the maximal number of positive components among all optimal solutions of problem $(\mathrm{P})$ and satisfy

$$
\sum_{x_{i}^{*}>0} \frac{\left(w_{i}\right)^{1 / \gamma_{2}}\left(x_{i}^{*}-\bar{x}_{i}\right)}{\bar{x}_{i}^{\gamma_{1} / \gamma_{2}}} \leq 0
$$

Since function $-a^{1-\frac{\gamma_{1}}{\gamma_{2}}}$ is strictly convex for $a>0$, from Lemma 2.9, we have for each $i$ with $x_{i}^{*}>0$,

$$
\left(x_{i}^{*}-\bar{x}_{i}\right)\left(-\left(1-\frac{\gamma_{1}}{\gamma_{2}}\right)\left(\bar{x}_{i}\right)^{-\frac{\gamma_{1}}{\gamma_{2}}}\right) \leq-\left(x_{i}^{*}\right)^{1-\frac{\gamma_{1}}{\gamma_{2}}}+\left(\bar{x}_{i}\right)^{1-\frac{\gamma_{1}}{\gamma_{2}}}
$$

hence

$$
\begin{aligned}
& \sum_{x_{i}^{*}>0}\left(w_{i}\right)^{1 / \gamma_{2}}\left(x_{i}^{*}\right)^{1-\frac{\gamma_{1}}{\gamma_{2}}}-\sum_{x_{i}^{*}>0}\left(w_{i}\right)^{1 / \gamma_{2}}\left(\bar{x}_{i}\right)^{1-\frac{\gamma_{1}}{\gamma_{2}}} \\
\leq & \left(1-\frac{\gamma_{1}}{\gamma_{2}}\right) \sum_{x_{i}^{*}>0} \frac{\left(w_{i}\right)^{1 / \gamma_{2}}\left(x_{i}^{*}-\bar{x}_{i}\right)}{\bar{x}_{i}^{\gamma_{1} / \gamma_{2}}} \leq 0 .
\end{aligned}
$$

Therefore $\bar{x}$ is the analytic center of the optimal solution set of problem (P) corresponding to function $-\sum_{x_{i}^{*}>0}\left(w_{i}\right)^{1 / \gamma_{2}}\left(x_{i}\right)^{1-\frac{\gamma_{1}}{\gamma_{2}}}$.

b) If $\gamma_{1}=\gamma_{2}$, and there exist a pair of primal and dual optimal solutions satisfying the strict complementarity, we know that $x^{*}$ and $z^{*}$ satisfy the strict complementarity. Since $\left(y^{*}, z^{*}\right)$ is an optimal solution for problem (D), we have

$$
\begin{aligned}
f\left(x^{*}\right)=L\left(y^{*}, z^{*}\right) & =\inf _{x \in R^{n}} f(x)+(b-A x)^{T} y^{*}-x^{T} z^{*} \\
& \leq f\left(x^{*}\right)+\left(b-A x^{*}\right)^{T} y^{*}-\left(x^{*}\right)^{T} z^{*}=f\left(x^{*}\right)-\left(x^{*}\right)^{T} z^{*} \leq f\left(x^{*}\right),
\end{aligned}
$$

hence

$$
\left(x^{*}\right)^{T} z^{*}=0
$$


and

$$
\inf _{x \in R^{n}} f(x)+(b-A x)^{T} y^{*}-x^{T} z^{*}=f\left(x^{*}\right)+\left(b-A x^{*}\right)^{T} y^{*}-\left(x^{*}\right)^{T} z^{*},
$$

which indicates

$$
A^{T} y^{*}+z^{*}=\nabla f\left(x^{*}\right)
$$

Therefore from Theorem 2.4, we have

$$
\begin{aligned}
\left(x(t)-x^{*}\right)^{T}\left(z(t)-z^{*}\right) & =\left(x-x^{*}\right)^{T}\left(A^{T}\left(y^{*}-y\right)+\nabla f(x)-\nabla f\left(x^{*}\right)\right) \\
& =\left(x-x^{*}\right)^{T}\left(\nabla f(x)-\nabla f\left(x^{*}\right)\right) \geq 0,
\end{aligned}
$$

or

$$
\sum_{x_{i}^{*}>0}\left(x_{i}^{*} z(t)_{i}\right)+\sum_{z_{i}^{*}>0} z_{i}^{*} x(t)_{i} \leq x(t)^{T} z(t)
$$

By using (7), we can get

$$
\sum_{x_{i}^{*}>0} \frac{x_{i}^{*}}{x(t)_{i}}\left(w_{i} \mu\left(1+d_{i}^{0}(t)\right)\right)^{\frac{1}{\gamma_{1}}}+\sum_{z_{i}^{*}>0} \frac{z_{i}^{*}}{z(t)_{i}}\left(w_{i} \mu\left(1+d_{i}^{0}(t)\right)\right)^{\frac{1}{\gamma_{1}}} \leq \sum_{i=1}^{n}\left(w_{i} \mu\left(1+d_{i}^{0}(t)\right)\right)^{\frac{1}{\gamma_{1}}},
$$

or

$$
\sum_{x_{i}^{*}>0} \frac{x_{i}^{*}}{x(t)_{i}}\left(w_{i}\left(1+d_{i}^{0}(t)\right)\right)^{\frac{1}{\gamma_{1}}}+\sum_{z_{i}^{*}>0} \frac{z_{i}^{*}}{z(t)_{i}}\left(w_{i}\left(1+d_{i}^{0}(t)\right)\right)^{\frac{1}{\gamma_{1}}} \leq \sum_{i=1}^{n}\left(w_{i}\left(1+d_{i}^{0}(t)\right)\right)^{\frac{1}{\gamma_{1}}},
$$

hence for any point $(\bar{x}, \bar{y}, \bar{z}) \in \Omega^{1}\left(x^{0}, y^{0}, z^{0}\right), \bar{x}$ and $\bar{z}$ also have the maximal numbers of positive components among all optimal solutions and satisfy the strict complementarity, so we must have

$$
\sum_{x_{i}^{*}>0} \frac{x_{i}^{*}}{\bar{x}_{i}} \frac{w_{i}^{1 / \gamma_{1}}}{\sum_{i=1}^{n} w_{i}^{1 / \gamma_{1}}}+\sum_{z_{i}^{*}>0} \frac{z_{i}^{*}}{\bar{z}_{i}} \frac{w_{i}^{1 / \gamma_{1}}}{\sum_{i=1}^{n} w_{i}^{1 / \gamma_{1}}} \leq 1
$$

and

$$
\sum_{x_{i}^{*}>0} \frac{w_{i}^{1 / \gamma_{1}}}{\sum_{i=1}^{n} w_{i}^{1 / \gamma_{1}}}+\sum_{z_{i}^{*}>0} \frac{w_{i}^{1 / \gamma_{1}}}{\sum_{i=1}^{n} w_{i}^{1 / \gamma_{1}}}=1
$$

Therefore

$$
e^{\sum_{x_{i}^{*}>0} \frac{w_{i}^{1 / \gamma_{1}}}{\sum_{i=1}^{n} w_{i}^{1 / \gamma_{1}}} \ln \frac{x_{i}^{*}}{\bar{x}_{i}}+\sum_{z_{i}^{*}>0} \frac{w_{i}^{1 / \gamma_{1}}}{\sum_{i=1}^{n} w_{i}^{1 / \gamma_{1}}} \ln \frac{z_{i}^{*}}{\bar{z}_{i}}} \leq e^{\ln \sum_{x_{i}^{*}>0} \frac{x_{i}^{*}}{\bar{x}_{i}} \frac{w_{i}^{1 / \gamma_{1}}}{\sum_{i=1}^{n} w_{i}^{1 / \gamma_{1}}}+\sum_{z_{i}^{*}>0} \frac{z_{i}^{*}}{\bar{z}_{i}} \frac{w_{i}^{1 / \gamma_{1}}}{\sum_{i=1}^{n} w_{i}^{1 / \gamma_{1}}}} \leq 1
$$


which implies

$$
\left(\prod_{x_{i}^{*}>0}\left(\frac{x_{i}^{*}}{\bar{x}_{i}}\right)^{w_{i}^{1 / \gamma_{1}}}\right)\left(\prod_{z_{i}^{*}>0}\left(\frac{z_{i}^{*}}{\bar{z}_{i}}\right)^{w_{i}^{1 / \gamma_{1}}}\right) \leq 1
$$

or

$$
\left(\prod_{x_{i}^{*}>0}\left(x_{i}^{*}\right)^{w_{i}^{1 / \gamma_{1}}}\right)\left(\prod_{z_{i}^{*}>0}\left(z_{i}^{*}\right)^{w_{i}^{1 / \gamma_{1}}}\right) \leq\left(\prod_{x_{i}^{*}>0}\left(\bar{x}_{i}\right)^{w_{i}^{1 / \gamma_{1}}}\right)\left(\prod_{z_{i}^{*}>0}\left(\bar{z}_{i}\right)^{w_{i}^{1 / \gamma_{1}}}\right) .
$$

Therefore $\bar{x}$ is the analytic center of the optimal solution set of problem (P) corresponding to function $-\prod_{x_{i}^{*}>0}\left(x_{i}\right)^{w_{i}^{1 / \gamma_{1}}}$.

If $\gamma_{1}=\gamma_{2}$ and $f(x)$ is analytic, the proof is contained in the proof of $\mathrm{c}$ ).

c) If $f(x)$ is analytic, then for any accumulation point $\bar{x}$ of $x(t)$ with $\bar{x} \neq x^{*}$, from Theorem 2.8 and Lemma 2.10, we have

$$
\left(x^{*}-\bar{x}\right)^{T} \nabla f(x)=0,
$$

for any $x \in R^{n}$. Hence from Theorem 2.4 and (7), we have

$$
\begin{aligned}
0 & =\left(x^{*}-\bar{x}\right)^{T} \nabla f(x(t))=\left(x^{*}-\bar{x}\right)^{T}\left(A^{T} y(t)+z(t)\right) \\
& =\left(x^{*}-\bar{x}\right)^{T} z(t)=\sum_{i=1}^{n} \frac{\left(w_{i} \mu\left(1+c_{i}^{0} e^{-\sigma\left(t-t_{0}\right)}\right)\right)^{1 / \gamma_{2}}\left(x_{i}^{*}-\bar{x}_{i}\right)}{x(t)_{i}^{\gamma_{1} / \gamma_{2}}}
\end{aligned}
$$

Similarly, we know that $\bar{x}$ must have the maximal number of positive components among all optimal solutions of problem (P) and satisfy

$$
\sum_{x_{i}^{*}>0} \frac{\left(w_{i}\right)^{1 / \gamma_{2}}\left(x_{i}^{*}-\bar{x}_{i}\right)}{\bar{x}_{i}^{\gamma_{1} / \gamma_{2}}}=0
$$

If $\gamma_{1}=\gamma_{2}$, from (12), we have

$$
\sum_{x_{i}^{*}>0} \frac{\left(w_{i}\right)^{1 / \gamma_{2}}}{\sum_{x_{i}^{*}>0}\left(w_{i}\right)^{1 / \gamma_{2}}} \frac{x_{i}^{*}}{\bar{x}_{i}}=1
$$

which implies

$$
e^{\sum_{x_{i}^{*}>0} \frac{w_{i}^{1 / \gamma_{1}}}{\sum_{x_{i}^{*}>0}^{\sum_{i}^{1 / \gamma_{1}}} \ln \frac{x_{i}^{*}}{\bar{x}_{i}}}} \leq e^{\ln \sum_{x_{i}^{*}>0} \frac{x_{i}^{*}}{\bar{x}_{i}} \frac{w_{i}^{1 / \gamma_{1}}}{\sum_{x_{i}^{*}>0} w_{i}^{1 / \gamma_{1}}}}=1
$$

thus

$$
\left(\prod_{x_{i}^{*}>0}\left(\frac{x_{i}^{*}}{\bar{x}_{i}}\right)^{w_{i}^{1 / \gamma_{1}}}\right) \leq 1 \quad \text { or } \quad\left(\prod_{x_{i}^{*}>0}\left(x_{i}^{*}\right)^{w_{i}^{1 / \gamma_{1}}}\right) \leq\left(\prod_{x_{i}^{*}>0}\left(\bar{x}_{i}\right)^{w_{i}^{1 / \gamma_{1}}}\right) .
$$


Therefore $\bar{x}$ is the analytic center of the optimal solution set of problem (P) corresponding to function $-\prod_{x_{i}^{*}>0}\left(x_{i}\right)^{w_{i}^{1 / \gamma_{1}}}$.

If $\gamma_{1}>\gamma_{2}$, since the function $a^{1-\frac{\gamma_{1}}{\gamma_{2}}}$ is strictly convex for $a>0$, from Lemma 2.9, we have for every $i$ such that $x_{i}^{*}>0$,

$$
\left(x_{i}^{*}-\bar{x}_{i}\right)\left[\left(1-\frac{\gamma_{1}}{\gamma_{2}}\right)\left(\bar{x}_{i}\right)^{-\frac{\gamma_{1}}{\gamma_{2}}}\right] \leq\left(x_{i}^{*}\right)^{1-\frac{\gamma_{1}}{\gamma_{2}}}-\left(\bar{x}_{i}\right)^{1-\frac{\gamma_{1}}{\gamma_{2}}}
$$

so from (12),

$$
\begin{aligned}
& \sum_{x_{i}^{*}>0}\left(w_{i}\right)^{1 / \gamma_{2}}\left(x_{i}^{*}\right)^{1-\frac{\gamma_{1}}{\gamma_{2}}}-\sum_{x_{i}^{*}>0}\left(w_{i}\right)^{1 / \gamma_{2}}\left(\bar{x}_{i}\right)^{1-\frac{\gamma_{1}}{\gamma_{2}}} \\
\geq & \left(1-\frac{\gamma_{1}}{\gamma_{2}}\right) \sum_{x_{i}^{*}>0} \frac{\left(w_{i}\right)^{1 / \gamma_{2}}\left(x_{i}^{*}-\bar{x}_{i}\right)}{\bar{x}_{i}^{\gamma_{1} / \gamma_{2}}}=0 .
\end{aligned}
$$

Therefore $\bar{x}$ is the analytic center of the optimal solution set of problem (P) corresponding to function $\sum_{x_{i}^{*}>0}\left(w_{i}\right)^{1 / \gamma_{2}}\left(x_{i}\right)^{1-\frac{\gamma_{1}}{\gamma_{2}}}$.

Proof of (iii). If $z^{*}=0$, the result is evident, so we assume $z^{*} \neq 0$ below. For any point $(\bar{x}, \bar{y}, \bar{z}) \in \Omega^{1}\left(x^{0}, y^{0}, z^{0}\right)$, if $\bar{z} \neq z^{*}$, from Theorem 2.8 , same as the analysis for (10) and (11), we can get

$$
A^{T} \bar{y}+\bar{z}=\nabla f(\bar{x})=\nabla f\left(x^{*}\right)
$$

and

$$
L\left(y^{*}, z^{*}\right)=f\left(x^{*}\right)-\nabla f\left(x^{*}\right)^{T} x^{*}+b^{T} y^{*}=L(\bar{y}, \bar{z})=f\left(x^{*}\right)-\nabla f\left(x^{*}\right)^{T} x^{*}+b^{T} \bar{y},
$$

which implies

$$
b^{T} \bar{y}=b^{T} y^{*}
$$

Since $z^{*}$ has the maximal number of positive components among all optimal solutions of problem (D), so from Theorem 2.8, for any $i$ with $z_{i}^{*}=0, \bar{z}_{i}=0$. Hence from (7), (13), and (14),

$$
\begin{aligned}
\sum_{z_{i}^{*}>0} \frac{\left(w_{i} \mu\left(1+d_{i}^{0}(t)\right)\right)^{1 / \gamma_{1}}\left(z_{i}^{*}-\bar{z}_{i}\right)}{z(t)_{i}^{\gamma_{2} / \gamma_{1}}} & =x(t)^{T}\left(z^{*}-\bar{z}\right)=x(t)^{T}\left(A^{T} \bar{y}-A^{T} y^{*}\right) \\
& =b^{T} \bar{y}-b^{T} y^{*}=0 .
\end{aligned}
$$

Thus

$$
\sum_{z_{i}^{*}>0} \frac{\left(w_{i}\left(1+d_{i}^{0}(t)\right)\right)^{1 / \gamma_{1}}\left(z_{i}^{*}-\bar{z}_{i}\right)}{z(t)_{i}^{\gamma_{2} / \gamma_{1}}}=0
$$

which indicates that $\bar{z}$ must have the maximal number of positive components 
among all optimal solutions of problem (D) and satisfy

$$
\sum_{z_{i}^{*}>0} \frac{\left(w_{i}\right)^{1 / \gamma_{1}}\left(z_{i}^{*}-\bar{z}_{i}\right)}{\bar{z}_{i}^{\gamma_{2} / \gamma_{1}}}=0 .
$$

Therefore, similar to the claim in the proof of (ii), we know if $\gamma_{1}<\gamma_{2}, \bar{z}$ is the analytic center of the optimal solution set corresponding to $\sum_{z_{i}^{*}>0}\left(w_{i}\right)^{1 / \gamma_{1}}\left(z_{i}\right)^{1-\frac{\gamma_{2}}{\gamma_{1}}}$. If $\gamma_{1}=\gamma_{2}, \bar{z}$ is the analytic center of the optimal solution set corresponding to $-\prod_{z_{i}^{*}>0}\left(z_{i}\right)^{w_{i}^{1 / \gamma_{1}}}$. If $\gamma_{1}>\gamma_{2}, \bar{z}$ is the analytic center of the optimal solution set corresponding to $-\sum_{z_{i}^{*}>0}\left(w_{i}\right)^{1 / \gamma_{1}}\left(z_{i}\right)^{1-\frac{\gamma_{2}}{\gamma_{1}}}$. Thus the theorem is proved.

\section{The extended primal-dual affine scaling continuous trajectory}

In this section, we study the extended primal-dual affine scaling continuous trajectory which is defined by the solution curve of the following ODE system

$$
\left\{\begin{array}{l}
-\nabla^{2} f(x) \frac{d x}{d t}+A^{T} \frac{d y}{d t}+\frac{d z}{d t}=0 \\
A \frac{d x}{d t}=0 \\
\gamma_{1} X^{\gamma_{1}-1} Z^{\gamma_{2}} \frac{d x}{d t}+\gamma_{2} X^{\gamma_{1}} Z^{\gamma_{2}-1} \frac{d z}{d t}=-X^{\gamma_{1}} Z^{\gamma_{2}} e \\
\left(x\left(t_{0}\right), y\left(t_{0}\right), z\left(t_{0}\right)=\left(x^{0}, y^{0}, z^{0}\right) \in \mathcal{F}^{0}\right.
\end{array}\right.
$$

where

$$
\begin{aligned}
& t_{0}>0, \mu=\frac{e^{T} X^{\gamma_{1}} Z^{\gamma_{2}} e}{n}, \gamma_{1}>0, \gamma_{2}>0, x \in R_{++}^{n}, X=\operatorname{diag}(x) \in R^{n \times n}, \\
& z \in R_{++}^{n}, Z=\operatorname{diag}(z) \in R^{n \times n} .
\end{aligned}
$$

Note: ODE system (15) is just ODE system (2) with $\sigma=0$. We call this ODE system the extended primal-dual affine scaling ODE system. The unique solution of ODE system (15) defines the extended primal-dual affine scaling continuous trajectory for problem (P). Similar to the weighted primal-dual path-following ODE system (2), this ODE system also has two explicit forms which are same as that of ODE system (2) but with $\sigma=0$. The following assumption is made throughout Section 3.

Assumption 2 The set $\mathcal{F}^{0}$ is nonempty.

\subsection{Properties of the extended primal-dual affine scaling continuous trajectory}

The results of Theorems 2.3, 2.4, 2.5, and 2.6 in Section 2 also hold for this extended primal-dual affine scaling ODE system (15), and the proofs are almost the same, hence we omit them. Now we can define the limit set for the unique solution $(x(t), y(t), z(t))$ of the extended primal-dual affine scaling ODE system (15) as well, which is denoted by $\Omega^{2}\left(x^{0}, y^{0}, z^{0}\right)$, and have the following similar result.

Theorem 3.1 The limit set $\Omega^{2}\left(x^{0}, y^{0}, z^{0}\right)$ is nonempty, compact, and connected. Furthermore $\Omega^{2}\left(x^{0}, y^{0}, z^{0}\right)$ is contained in $\mathcal{F}$. 


\subsection{Optimality of the cluster point(s)}

In this subsection, we just show that every accumulation point of the solution of the extended primal-dual affine scaling ODE system (15) is an optimal solution for problems (P) and (D).

THEOREM 3.2 Let $(x(t), y(t), z(t))$ be the unique solution of the extended primaldual affine scaling ODE system (15). Then

(i) $x(t)^{T} z(t) \leq L_{3} e^{-\left(t-t_{0}\right) / \max \left(\gamma_{1}, \gamma_{2}\right)}$, where $L_{3}>0$ is a constant which depends only on $x^{0}, z^{0}, \gamma_{1}$, and $\gamma_{2}$, hence for any point $\left(x^{1}, y^{1}, z^{1}\right) \in \Omega^{2}\left(x^{0}, y^{0}, z^{0}\right)$, $x^{1}$ is an optimal solution of problem $(P),\left(y^{1}, z^{1}\right)$ is an optimal solution of problem (D).

(ii) For any $1 \leq i \leq n$,

$$
\left(x(t)_{i}^{\gamma_{1}} z(t)_{i}^{\gamma_{2}}-\mu\right)=e^{-\left(t-t_{0}\right)}\left(x\left(t_{0}\right)_{i}^{\gamma_{1}} z\left(t_{0}\right)_{i}^{\gamma_{2}}-\mu^{0}\right),
$$

where $\mu^{0}=\frac{e^{T} X\left(t_{0}\right)^{\gamma_{1}} Z\left(t_{0}\right)^{\gamma_{2}} e}{n}$.

Proof. The proof is similar to the proof of Theorem 2.8.

\subsection{Convergence of the extended primal-dual affine scaling continuous trajectory}

Now, it comes to the key result of Section 3. Theorem 3.3 below shows that the solution of the extended primal-dual affine scaling ODE system (15) converges to the analytic centers of the optimal solution sets corresponding to some convex functions which depend on the initial point as $t \rightarrow+\infty$ and is actually a weighted central path. Similar to Theorem 2.11, we let $x^{*}$ and $\left(y^{*}, z^{*}\right)$ be optimal solutions for problems $(\mathrm{P})$ and $(\mathrm{D})$, respectively, such that $x^{*}$ and $z^{*}$ have the maximal numbers of positive components among all optimal solutions.

TheOREm 3.3 Let $(x(t), y(t), z(t))$ be the unique solution of the extended primaldual affine scaling ODE system (15), $c_{i}^{0}=\frac{\left(x_{i}^{0}\right)^{\gamma_{1}}\left(z_{i}^{0}\right)^{\gamma_{2}}}{\mu^{0}}$ and $\mu^{0}=\frac{e^{T} X\left(t_{0}\right)^{\gamma_{1}} Z\left(t_{0}\right)^{\gamma_{2}} e}{n}$. Then

(i) for any $1 \leq i \leq n$,

$$
x(t)_{i}^{\gamma_{1}} z(t)_{i}^{\gamma_{2}}=\frac{x\left(t_{0}\right)_{i}^{\gamma_{1}} z\left(t_{0}\right)_{i}^{\gamma_{2}}}{\mu_{0}} \mu .
$$

(ii) The same as (ii) of Theorem 2.11 but with $w_{i}=c_{i}^{0}$.

(iii) The same as (iii) of Theorem 2.11 but with $w_{i}=c_{i}^{0}$.

Proof. The proof is similar to the proof of Theorem 2.11.

\section{The weighted central path family}

In Section 2, the weighted primal-dual path-following continuous trajectory (2) contains the weighted central path if the initial point is on the central path. The 
weighted central path actually comes from the following equation system:

$$
\left(\begin{array}{c}
A^{T} y+z \\
A x \\
X^{\gamma_{1}} Z^{\gamma_{2}} e
\end{array}\right)=\left(\begin{array}{c}
\nabla f(x) \\
b \\
\mu w
\end{array}\right)
$$

where $x>0, z>0, \mu>0$ is the parameter and $w \in R_{++}^{n}$ is a given weighted vector. Taking derivative with respect to $\mu$ in the above equation, we can get

$$
\frac{d x}{d \mu}=\frac{1}{\gamma_{1} \mu}\left[I+\frac{\gamma_{2}}{\gamma_{1}} D P_{A D} D \nabla^{2} f(x)\right]^{-1} D P_{A D} D \nabla f(x),
$$

where $D=W^{-\frac{1}{2 \gamma_{2}}} X^{\frac{\gamma_{1}+\gamma_{2}}{2 \gamma_{2}}}$ and $P_{A D}=I-D A^{T}\left(A D^{2} A^{T}\right)^{-1} A D$. Let $t=\mu^{-\frac{1}{\gamma_{2}}}$. Then the above ODE system becomes

$$
\frac{d x}{d t}=-\left[\frac{\gamma_{1}}{\gamma_{2}} I+t D P_{A D} D \nabla^{2} f(x)\right]^{-1} D P_{A D} D \nabla f(x) .
$$

If we do not require the initial point to be on the central path, we can get the following ODE system

$$
\frac{d x}{d t}=-\left[\frac{\gamma_{1}}{\gamma_{2}} I+t D P_{A D} D \nabla^{2} f(x)\right]^{-1} D P_{A D} D \nabla f(x), x\left(t_{0}\right)=x^{0} \in \mathcal{P}^{++},
$$

where

$$
\begin{aligned}
& t_{0}>0, \gamma_{1}>0, \gamma_{2}>0, x \in R_{++}^{n}, X=\operatorname{diag}(x) \in R^{n \times n}, \\
& w \in R_{++}^{n}, W=\operatorname{diag}(w), D=W^{-\frac{1}{2 \gamma_{2}}} X^{\frac{\gamma_{1}+\gamma_{2}}{2 \gamma_{2}}} .
\end{aligned}
$$

We call this ODE system the weighted central path family ODE system. The unique solution of ODE system (16) defines the weighted central path family (a continuous trajectory) for problem (P). For ODE system (16), we sometimes use its equivalent implicit form

$$
\frac{d x}{d t}=-\frac{\gamma_{2}}{\gamma_{1}} D P_{A D} D\left[\nabla f(x)+t \nabla^{2} f(x) \frac{d x}{d t}\right], x\left(t_{0}\right)=x^{0} \in \mathcal{P}^{++} .
$$

The following assumptions are made throughout Section 4 .

Assumption 3 The optimal solution set of problem $(\mathrm{P})$ is non-empty and bounded.

Assumption 4 The set $\mathcal{P}^{++}$is not empty.

\subsection{Properties of the weighted central path family}

Similar to Theorem 2.3, there exists a unique solution $x(t)$ on the maximal existence interval for this weighted central path family ODE system (16). In the linear case, the weighted central path family is actually the weighted affine scaling continuous trajectory [20]. In [20], Adler and Monteiro used some auxiliary optimization problems and auxiliary continuous trajectories $y(\mu)$ and $z(\mu)$ to study the limiting behavior of the affine scaling continuous trajectories. Here we also adopt the 
same strategy. In order to propose the auxiliary optimization problems, we choose a $y^{0} \in R^{m}$, and let $z^{0}=\nabla f\left(x^{0}\right)-A^{T} y^{0}, p=t_{0} z^{0}-X\left(t_{0}\right)^{-\frac{\gamma_{1}}{\gamma_{2}}} W^{\frac{1}{\gamma_{2}}} e$. Then we get the following lemma.

Lemma 4.1 For any $t \geq t_{0}$, the following optimization problem

$$
\begin{array}{ll}
\min & f(x)-\frac{1}{t}\left(p^{T} x+\sum_{i=1}^{n} q_{i}(x) w_{i}^{\frac{1}{\gamma_{2}}}\right) \\
\text { s.t. } & A x=b, x>0
\end{array}
$$

where

$$
q_{i}(x)= \begin{cases}\frac{\gamma_{2}}{\gamma_{2}-\gamma_{1}} x_{i}^{1-\frac{\gamma_{1}}{\gamma_{2}}} & \text { if } \gamma_{1} \neq \gamma_{2}, \\ \ln x_{i} & \text { if } \gamma_{1}=\gamma_{2},\end{cases}
$$

has a unique optimal solution, and all optimial solutions are contained in a bounded set.

Proof. For $t=t_{0}$, this is evident since $\left(x^{0}, y^{0}, z^{0}\right)$ satisfies the following KKT system

$$
\left\{\begin{array}{l}
A^{T} y^{0}+z^{0}=\nabla f\left(x^{0}\right) \\
A x^{0}=b, x^{0}>0 \\
t_{0} z^{0}=X\left(t_{0}\right)^{-\frac{\gamma_{1}}{\gamma_{2}}} W^{\frac{1}{\gamma_{2}}} e+p
\end{array}\right.
$$

Since for $t=t_{0}$, the objective function is strictly convex, implying that the optimal solution set is a single point $\left(x^{0}, y^{0}, z^{0}\right)$ which must be bounded. Hence from Theorem 24 in [41], the level set will be of course bounded as well. For any $\alpha>0$, $t_{0}<t<3 t_{0}$ and $x \in \mathcal{P}^{++}$, if

$$
f(x)-\frac{1}{t}\left(p^{T} x+\sum_{i=1}^{n} q_{i}(x) w_{i}^{\frac{1}{\gamma_{2}}}\right) \leq \alpha,
$$

then

$$
\begin{aligned}
f(x)-\frac{1}{t_{0}}\left(p^{T} x+\sum_{i=1}^{n} q_{i}(x) w_{i}^{\frac{1}{\gamma_{2}}}\right) & \leq \frac{t}{t_{0}} \alpha-\left(\frac{t}{t_{0}}-1\right) f(x) \\
& \leq \frac{t}{t_{0}} \alpha-\left(\frac{t}{t_{0}}-1\right) f\left(x^{*}\right) \leq 3 \alpha+2\left|f\left(x^{*}\right)\right|,
\end{aligned}
$$

where $x^{*}$ is an optimal solution for problem $(\mathrm{P})$. The above inequality implies that $x$ is bounded, hence the level set for $t_{0}<t<3 t_{0}$ is bounded. For any $\alpha>0, t>2 t_{0}$ and $x \in \mathcal{P}^{++}$, if

$$
f(x)-\frac{1}{t}\left(p^{T} x+\sum_{i=1}^{n} q_{i}(x) w_{i}^{\frac{1}{\gamma_{2}}}\right) \leq \alpha,
$$


then

$$
\begin{aligned}
f(x) & \leq \frac{t}{t-t_{0}} \alpha-\frac{t_{0}}{t-t_{0}}\left(f(x)-\frac{1}{t_{0}}\left(p^{T} x+\sum_{i=1}^{n} q_{i}(x) w_{i}^{\frac{1}{\gamma_{2}}}\right)\right) \\
& \leq 2 \alpha-\frac{t_{0}}{t-t_{0}}\left(f\left(x^{0}\right)-\frac{1}{t_{0}}\left(p^{T} x^{0}+\sum_{i=1}^{n} q_{i}\left(x^{0}\right) w_{i}^{\frac{1}{\gamma_{2}}}\right)\right) \\
& \leq 2 \alpha+2\left|f\left(x^{0}\right)-\frac{1}{t_{0}}\left(p^{T} x^{0}+\sum_{i=1}^{n} q_{i}\left(x^{0}\right) w_{i}^{\frac{1}{\gamma_{2}}}\right)\right|
\end{aligned}
$$

which implies that $x$ is bounded since the level set for problem $(\mathrm{P})$ is bounded under Assumption 3, hence the level set for $t>2 t_{0}$ is also bounded. Therefore for any $t \geq t_{0}$, the level set for problem $(\mathrm{Pt})$ is bounded, and the bound is independent of $t$. Since the objective function for problem (Pt) is strictly convex for any $t \geq t_{0}$, it has a unique optimal solution, moreover all optimal solutions are contained in a bounded set.

From Lemma 4.1, we can get the auxiliary continuous trajectories $y(t)$ and $z(t)$.

THEOREM 4.2 There exist two auxiliary continuous trajectories $y(t)$ and $z(t)$ for $t \geq t_{0}$ such that $(x(t), y(t), z(t))$ satisfies the following system

$$
\left\{\begin{array}{l}
A^{T} y(t)+z(t)=\nabla f(x(t)) \\
A x(t)=b, x(t)>0 \\
t z(t)=X(t)^{-\frac{\gamma_{1}}{\gamma_{2}}} W^{\frac{1}{\gamma_{2}}} e+p
\end{array}\right.
$$

where $x(t)$ is the unique solution of the weighted central path family ODE system (16).

Proof. From Lemma 4.1, for any $t \geq t_{0}$, there exists a unique solution for system (19). Since $f(x) \in C^{3}$ on $R^{n}$, we can take derivative of system (19) with respect to $t$ and get the $\frac{d x}{d t}$ which is actually the right-hand side of the weighted central path family ODE system (16). Hence the unique solution $x(t)$ of system (19) is actually the unique solution of ODE system (16).

Theorem 4.2 also shows the existence of $x(t)$ for any $t \geq t_{0}$, i.e., the maximal existence interval of $x(t)$ in (16) is $\left[t_{0},+\infty\right)$. Next we show that $y(t)$ and $z(t)$ are also bounded.

TheOREM 4.3 The auxiliary continuous trajectories $y(t)$ and $z(t)$ are bounded.

Proof. From system (19), we have

$$
X^{\frac{\gamma_{1}}{\gamma_{2}}} z=\frac{1}{t}\left[W^{\frac{1}{\gamma_{2}}} e+X^{\frac{\gamma_{1}}{\gamma_{2}}} p\right]
$$

since $x(t)$ is bounded, we know $x(t)_{i}^{\gamma_{1}} z(t)_{i}^{\gamma_{2}} \rightarrow 0$ as $t \rightarrow+\infty$. Then similar to the proof of Theorem 2.5, we can prove $y(t)$ and $z(t)$ are bounded as well.

Now we can define the limit set for the unique solution $(x(t), y(t), z(t))$ of system (19) as well, which is denoted by $\Omega^{3}\left(x^{0}, y^{0}, z^{0}\right)$, and have the following similar results. 
THEOREM 4.4 The limit set $\Omega^{3}\left(x^{0}, y^{0}, z^{0}\right)$ is nonempty, compact, and connected. Furthermore $\Omega^{3}\left(x^{0}, y^{0}, z^{0}\right)$ is contained in $\mathcal{F}$.

\subsection{Optimality of the cluster point(s)}

In this subsection, we just show that every point in $\Omega^{3}\left(x^{0}, y^{0}, z^{0}\right)$ is an optimal solution for problems (P) and (D).

TheORem 4.5 For any point $\left(x^{1}, y^{1}, z^{1}\right) \in \Omega^{3}\left(x^{0}, y^{0}, z^{0}\right), x^{1}$ is an optimal solution of problem $(P),\left(y^{1}, z^{1}\right)$ is an optimal solution of problem $(D)$.

Proof. From (20), we can get for any $1 \leq i \leq n,\left(x_{i}^{1}\right)^{\gamma_{1}}\left(z_{i}^{1}\right)^{\gamma_{2}}=0$, which indicates $x_{i}^{1} z_{i}^{1}=0$. Furthermore from system (19), we have $z(t)>\frac{p}{t}$ and $x(t)>0$, which imply $x^{1} \geq 0$ and $z^{1} \geq 0$. From system (19), we also have

$$
A^{T} y^{1}+z^{1}=\nabla f\left(x^{1}\right), A x^{1}=b
$$

Hence $\left(x^{1}, y^{1}, z^{1}\right)$ satisfies the KKT system for problems (P) and (D).

\subsection{Convergence of the weighted central path family}

Theorem 4.6 below shows that the solution of the weighted central path family ODE system (16) converges to the analytic centers of the optimal solution sets corresponding to some convex functions. Similar to Theorem 2.11, we let $x^{*}$ and $\left(y^{*}, z^{*}\right)$ be optimal solutions for problems (P) and (D), respectively, such that $x^{*}$ and $z^{*}$ have the maximal numbers of positive components among all optimal solutions.

Theorem 4.6 Let $(x(t), y(t), z(t))$ be the unique solution of system (19). Then

(i) a) if $\gamma_{1}<\gamma_{2}$, then $x(t)$ will converge to the analytic center of the optimal solution set of problem (P) corresponding to $-\left(\sum_{x_{i}^{*}>0} \frac{\gamma_{2}}{\gamma_{2}-\gamma_{1}}\left(w_{i}\right)^{1 / \gamma_{2}}\left(x_{i}\right)^{1-\frac{\gamma_{1}}{\gamma_{2}}}+p^{T} x\right) ;$

b) if $\gamma_{1}=\gamma_{2}$, and either there exists a pair of primal and dual optimal solutions satisfying the strict complementarity or $f(x)$ is analytic, then $x(t)$ will converge to the analytic center of the optimal solution set of problem $(P)$ corresponding to $-\left(\sum_{x_{i}^{*}>0}\left(w_{i}\right)^{1 / \gamma_{2}} \ln x_{i}+p^{T} x\right)$;

c) if $\gamma_{1}>\gamma_{2}$ and $f(x)$ is analytic, then $x(t)$ will converge to the analytic center of the optimal solution set of problem $(P)$ corresponding to

$$
-\left(\sum_{x_{i}^{*}>0} \frac{\gamma_{2}}{\gamma_{2}-\gamma_{1}}\left(w_{i}\right)^{1 / \gamma_{2}}\left(x_{i}\right)^{1-\frac{\gamma_{1}}{\gamma_{2}}}+p^{T} x\right) .
$$

(ii) The same as (iii) of Theorem 2.11. 
Proof. From system (19), we have

$$
x_{i}(t)=\frac{t^{-\frac{\gamma_{2}}{\gamma_{1}}} w_{i}^{\frac{1}{\gamma_{1}}}}{\left(z_{i}(t)-\frac{p_{i}}{t}\right)^{\frac{\gamma_{2}}{\gamma_{1}}}}>0,
$$

for any $i=1, \ldots, n$. Hence by using system (19), similar to the proof of Theorem 2.11, we can prove this theorem.

\section{Conclusions}

In this paper, three interior point continuous trajectories are fully studied for linearly constrained convex programming. Under some mild conditions and with proper choice of some parameters, all three continuous trajectories converge to some optimal solution(s) of convex programming without requiring either the strict complementarity or the analyticity of the objective function. Another very attractive feature for our three interior point continuous trajectories is that the initial point is only required to be in the interior of the feasible region. Therefore, many iterative solution schemes can be constructed based on these three interior point continuous trajectories for solving convex programming with general linear constraints. We will investigate these solution schemes in our future research.

\section{References}

[1] Monteiro, R. D. C. A globally convergent primal-dual interior point algorithm for convex programming. Math. Program. 1994; 64(1-3): 123-147

[2] Dikin, I. I. On the convergence of an iterative process. Upravlyaemye Sistemy. 1974; 12: 54-60 (in Russian)

[3] Saigal, R. A simple proof of a primal affine scaling method. Annals Oper. Res. 1996; 62: $303-324$

[4] Tseng, P., Luo, Z.-Q. On the convergence of the affine-scaling algorithm. Math. Program. 1992; 56: 301-319

[5] Tsuchiya, T. Global convergence of the affine scaling methods for degenerate linear programming problems. Math. Program. 1991; 52: 377-404

[6] Tsuchiya, T. Global convergence property of the affine scaling methods for primal degenerate linear programming problems. Math. Oper. Res. 1992; 17: 527-557

[7] Gonzaga, C. C., Carlos, L. A.:A primal affine-scaling algorithm for linearly constrained convex programs. 2002; http://www.optimizationonline.org/DB_HTML/2002/09/531.html.

[8] Sun, J. A convergence analysis for a convex version of Dikin's algorithm. Annals Oper. Res. 1996; 62: 357-374

[9] Tseng, P., Bomze, I. M., Schachinger, W. A first-order interior point method for linearly constrained smooth optimization. Math. Program. 2011; 127: 399-424

[10] Kojima, M., Mizuno, S., Yoshise, A. A primal-dual interior point algorithm for linear programming. Progress in Mathematical Programming. 1989; 61(1-3): 29-47

[11] Monteiro, R. D. C., Adler, I. Interior path-following primal-dual algorithm. Part I : Linear programming. Math. Program. 1989; 44(1-3): 27-41

[12] Monteiro, R. D. C., Adler, I. Interior path following primal-dual algorithms. part II: Convex quadratic programming. Math. Program. 1989; 44(1-3): 43-66

[13] Renegar, J. A polynomial-time algorithm based on Newton's method for linear programming. Math. Program. 1988; 40: 59-93 
14] Roos, C. New trajectory following polynomial-time algorithm for the linear programming problem. J. Optim. Theory Appl. 1989; 63(3): 433-458

[15] Todd, M. J., Ye, Y. A centered projective algorithm for linear programming. Math. Oper. Res. 1990; 15(3): 508-529

[16] Gonzaga, C. C. Path-following methods for linear programming. SIAM Rev. 1992; $34(2): 167-224$

[17] Zhu, J. A path following algorithm for a class of convex programming problems. ZORMethods and Models of Operations Research. 1992; 36(4): 359-377

[18] Monteiro, R. D. C., Adler, I., Resende, M. G. C. A polynomial-time primal-dual affine scaling algorithm for linear and convex quadratic programming and its power series extension. Math. Oper. Res. 1990; 15(2): 191-214

[19] Hertog, D. D., Roos, C. A survey of search directions in interior point methods for linear programming. Math. Program. 1991; 52(1-3): 481-509

[20] Adler, I., Monteiro, R. D. C. Limiting behavior of the affine scaling continuous trajectories for linear programming problems. Math. Program. 1991; 50: 29-51

[21] Bayer, D. A., Lagarias, J. C. The nonlinear geometry of linear programming. II Legendre transform coordinates and central trajectories. Trans. Am. Math. Soc. 1989; 314: $527-581$

[22] Liao, L.-Z. A study of the dual affine scaling continuous trajectories for linear programming. J. Optim. Theory Appl. 2014; 163: 548-568

[23] Megiddo, N., Shub, M. Boundary behavior of interior point algorihms for linear programming. Math. Oper. Res. 1989; 14: 97-146

[24] Monteiro, R. D. C. Convergence and bounary behavior of the projective scaling trajectories for linear programmin. Math. Oper. Res. 1991; 16: 842-858

[25] Monteiro, R. D. C. On the continuous trajectories for a potential reduction algorithm for linear programming. Math. Oper. Res. 1992; 17(1): 225-253

[26] Mclinden, L. An analogue of Moreau's proximation theorem, with application to the nonlinear complementarity problem. Pacific J. Math. 1980; 88(1): 101-161

[27] Megiddo, N. Pathways to the optimal set in linear programming. Progress in Mathematical Programming. 1989; 131-158

[28] Kojima, M., Mizuno, S., Noma, T. Limiting behavior of trajectories by a continuation method for monotone complementarity problems. Math. Oper. Res. 1990; 15: 662-675

[29] Monteiro, R. D. C., Tsuchiya, T. Limiting behavior of the derivatives of certain trajectories associated with a monotone horizontal linear complementarity problem. Math. Oper. Res. 1996; 21: 793-814

[30] Vavasis, S. A., Ye, Y. A primal-dual interior point method whose running time depends only on the constraint matrix. Math. Program. 1996; 74: 79-120

[31] Witzgall, C., Boggs, P. T., Domich, P. D. On the convergence behavior of trajectories for linear programming. Contemporary Mathematics. 1990; 161-188

[32] Monteiro, R. D. C., Zhou, F. J. On the existence and convergence of the central path for convex programming and some duality results. Comp. Optim. Appl. 1998; 10: 51-77

[33] Drummond, L. M. G., Svaiter, B. F. On well definedness of the central path. J. Optim. Theory Appl. 1999; 102: 223-237

[34] Gilbert, J. C., Gonzaga, C. C., Karas, E. Examples of ill-behaved central paths in convex optimization. Math. Program. 2005; 103: 63-94

[35] Wang, S. G., Wu, M. X., Jia, Z. Z. (ed.2) Matrix Inequalities. Beijing: Science Press; 2006 (in Chinese).

[36] Anosov, D. V., Aranson, S. Kh., Arnold, V. I., Bronshtein, I. U., Grines, V. Z., Il'yashenko, Yu. S. Ordinary Differential Equations and Smooth Dynamical Systems. Springer, 1988.

[37] Coddington, E. A., Levinson, N. Theory of Ordinary Diffenential Equations. New York: McGraw-Hill Book Co; 1955.

[38] Boyd, S., Vandenberghe, L. Convex Optimization. Cambridge University Press: Cambridge; 2004.

[39] Parks, H. R., Krantz, S. G. A primer of real analytic functions. Birkhäuser Verlag; 
1992.
[40] Rockafellar, R. T. Convex Analysis. Princeton Mathematical Series. No. 28. Princeton: Princeton University Press; 1970.
[41] Fiacco, A. V., McCormick, G. P. Nonlinear Programming: Sequential Unconstrained
Minimization Techniques. USA: SIAM. Philadelphia; 1990.

